
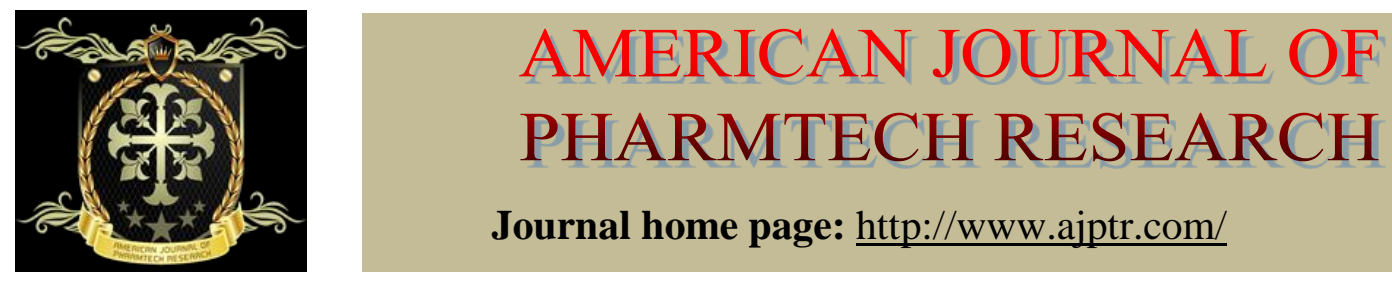

Journal home page: http://www.ajptr.com/

\title{
Development of Validated Bioanalytical RP-HPLC Method for Determination of Sofosbuvir In Human Plasma
}

\author{
S. Satheshkumar ${ }^{1 *}$, V. Muruganatham ${ }^{2}$ \\ 1. Ph.D Scholar, Department of Pharmaceutics, College of pharmacy, Vinayaka mission's \\ college of pharmacy, Salem - 636008, Tamilnadu, India. \\ 2. Professor, Department of Pharmaceutics, College of pharmacy, Vinayaka mission's college \\ of pharmacy, Salem - 636008, Tamilnadu, India.
}

\begin{abstract}
A linear, precise, accurate and simple reversed-phase high performance liquid chromatography (RPHPLC) bioanalytical method was developed and validated for determination of sofosbuvir in human plasma (K $\mathrm{K}_{2}$ EDTA) using ledipasvir as an internal standard. Sofosbuvir and ledipasvir (ISTD) were separated from plasma using solid phase extraction technique and The chromatographic separation was accomplished by using a Zorbax eclipse XDB C18 Column $(250 \times 4.6 \mathrm{~mm}$ i.d, $5 \mu \mathrm{m}$ particle size) with $1 \%$ orthophosphoric acid (pH6.4) and Acetonitrile in the ratio of $30: 70 \% \mathrm{v} / \mathrm{v}$, as mobile phase in an isocratic elution mode pumped at a flow rate $0.7 \mathrm{~mL} / \mathrm{min}$ and the column oven temperature was maintained at $25^{\circ} \mathrm{C}$. The wavelength selected for quantitation was $254 \mathrm{~nm}$. Retention times of sofosbuvir and internal standard were found to be $3.8 \mathrm{~min}$ and $7.4 \mathrm{~min}$, respectively. The standard curves were found to be linear in the range of 30.566-2000.381 ng/ml for sofosbuvir in human plasma. This method performed an intra-day and inter-day precision within the range of 2.44-11.14 and 5.01-8.70\%, respectively. Additional intra-day and inter-day accuracy was within the range of $87.99-105.93 \%$ and $94.97-99.18 \%$ respectively. Total percentage mean recovery of sofosbuvir from spiked plasma was found to be $86.54 \%$. All the validated parameters were found to be within the Acceptance limit.
\end{abstract}

Keywords: Human plasma, RP-HPLC, Sofosbuvir, Solid phase extraction.

*Corresponding Author Email: shaktipalpatil@yahoo.com

Received 15 February 2020, Accepted 29 February 2020

Please cite this article as: Satheshkumar S et al., Development of Validated Bioanalytical RP-HPLC Method for Determination of Sofosbuvir In Human Plasma . American Journal of PharmTech Research 2020. 


\section{INTRODUCTION}

Sofosbuvir (SOF) is a hepatitis $\mathrm{C}$ virus (HCV) non-structural protein (NS) 5B polymerase inhibitor. It was first approved for commercial marketing in the United States (US) on 06 December 2013 and in the European Union (EU) on 17 January 2014. Sofosbuvir is indicated for the treatment of genotypes 1 to 4 (US), and genotypes 1 to 6 (EU) HCV infection ${ }^{1}$. SOF is a prodrug and is extensively metabolized in the liver to form the pharmacologically active nucleoside analog triphosphate GS-461203. The active triphosphate GS-461203 was undetectable in plasma during both nonclinical and clinical studies. The two major metabolites are GS-331007 and GS-566500². Systematic (IUPAC) name: Isopropyl (2S)-2-[[[(2R,3R,4R,5R)-5-(2,4-dioxopyrimidin-1-yl)-4fluoro-3-hydroxy-4-methyl-tetrahydrofuran-yl] methoxy-phenoxy-phosphoryl]amino] propanoate (Figure 1$)^{3}$. It is freely soluble in Methanol and chemical formula is $\mathrm{C}_{22} \mathrm{H}_{29} \mathrm{FN}_{3} \mathrm{O}_{9} \mathrm{P}$ and the molecular weight is $529.458 \mathrm{~g} / \mathrm{mol}$.

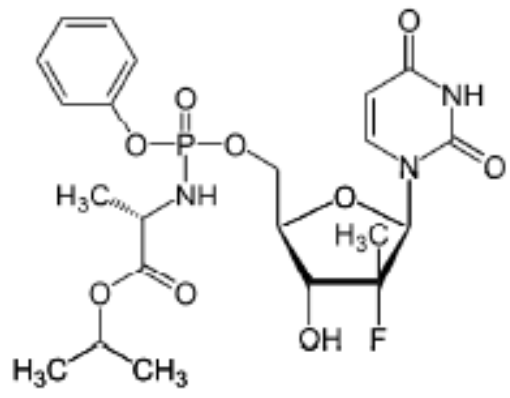

\section{Figure 1: Chemical structure of Sofosbuvir}

Literature review reveals that few analytical methods have been reported for the determination of sofosbuvir. which includes high performance liquid chromatography coupled with ultra violet (UV) detection and liquid chromatography-mass spectrometry (LC-MS) and ultra-high performance liquid chromatography tandem mass spectrometric detection (UPLC-MS/MS ${ }^{[4-13]}$. The present study was aimed to develop a novel, simple, economic and validated RP-HPLC method for the estimation of Sofosbuvir in human plasma according to USFDA guidelines ${ }^{14}$.

\section{MATERIALS AND METHOD}

Sofosbuvir and Ledipasvir bulk drug was kindly provided as gift sample by Sequent Labs Pvt. Ltd., Mangalore, India. Analytical grade of Ammonium acetate purchased from Rankem Ltd., India and HPLC grade of Methanol and Acetonitrile purchased from Merck Specialties Private Limited, India. Analytical grade Ortho phosphoric acid purchased from Rankem Ltd., India, Matrix: Human plasma ( $\mathrm{K}_{2}$ EDTA - Anticoagulant) obtained from a registered blood bank.

\section{Instrument}


Quantitative HPLC was performed on Shimadzu HPLC system with SPD-M10 A VP system PDA and LC-MS solution software. An XDB column of $250 \mathrm{~mm} 4.6 \mathrm{~mm}$ : i.d and $5 \mu$ particle size was used. Micro Weighing Balance (MX5) - Mettler Toledo, an Ultrasonic bath sonicator, Digital pH meter Mettler Toledo, Deep Freezer $\left(-20 \pm 5^{\circ} \mathrm{C}\right)$ - Thermo Fisher Scientific, Refrigerator $\left(2-8^{\circ} \mathrm{C}\right)$ - Thermo Fisher Scientific and Vortex - Spinix, Swirlex, Heidolph, Heidolph Reax top and Solid phase extraction(SPE) unit- Orochem Technologies Ezypress 48 were used in the study.

\section{Chromatographic parameters}

Equipment: Shimadzu HPLC system with SPD-M10 A VP system PDA detector

Column: Zorbax eclipse XDB C18 Column $(250 \times 4.6 \mathrm{~mm}, 5 \mu \mathrm{m}$

Mobile Phase: Acetonitrile and 1\% orthophosphoric acid (pH6.4) (70:30\% v/v)

Flow rate: $0.7 \mathrm{~mL} / \mathrm{min}$

Wavelength: $254 \mathrm{~nm}$

Injection volume: $20 \mu \mathrm{l}$

Run time: 10 Minutes

\section{Standard solution preparation}

Accurately weighed and transferred 5mg of Sofosbuvir into 5ml dry volumetric flask, The solution is made upto the mark using Methanol at a concentration of $1.0 \mathrm{mg} / \mathrm{mL}$ and stored at $2-8^{\circ} \mathrm{C}$.

\section{Working standard preparation}

The working solutions for the calibration standards containing sofosbuvir were prepared by dilution of the stock solution with the diluent $60 \%$ methanol solution (v/v). Calibration standards in human plasma were prepared by diluting the corresponding working solutions with blank plasma samples at the following concentrations: $30.300,60.600,120.905,250.048,500.095,1000.190,1500.286$ and $2000.381 \mathrm{ng} / \mathrm{mL}$ for sofosbuvir. The working solutions for the quality control (QC) samples were prepared at the following concentrations: 30.566, 80.376, 908.442 and $1700.417 \mathrm{ng} / \mathrm{mL}$ for sofosbuvir. IS working standard solution at a concentration of $500 \mathrm{ng} / \mathrm{mL}$ was prepared by diluting the stock solution $(1.0 \mathrm{mg} / \mathrm{mL})$ with the diluent $60 \%$ methanol solution $(\mathrm{v} / \mathrm{v})$. All stock solutions, working solutions, calibration standards and QCs were immediately stored at $2-8{ }^{\circ} \mathrm{C}$.

\section{Sample preparation:}

Exactly $0.400 \mathrm{~mL}$ of sample was aliquoted and transferred into a polypropylene tube and $50.00 \mu \mathrm{L}$ of ISTD solution was added, except for standard blank, to which $50.0 \mu \mathrm{L}$ of $60 \%$ methanol solution (v/v) was added and vortexed. To this $0.200 \mathrm{~mL}$ of $20 \mathrm{mM}$ ammonium acetate $(\mathrm{w} / \mathrm{v})$ was added and vortexed. Sample clean-up was conducted using the following SPE procedure, the cartridges (Oasis 
HLB) were first conditioned with methanol $1 \mathrm{~mL}$ then equilibrated by water $1 \mathrm{~mL}$, in a positive pressure manifold. Plasma samples were loaded onto SPE cartridges. Then, the cartridges were washed with water $1 \mathrm{~mL}$ twice and followed by $10 \%$ methanol solution $(\mathrm{v} / \mathrm{v}) 1 \mathrm{~mL}$ to wash the interferences and the analyte was successively eluted with $0.300 \mathrm{~mL}$ of methanol with elution Ria vial contained $50.00 \mu \mathrm{L}$ of $1 \%$ orthophosphoric acid (pH6.4) and vortexed. Finally $20 \mu \mathrm{L}$ was injected into the HPLC system.

\section{METHOD VALIDATION}

Method validation was done as per the criteria of industrial guidance for bioanalytical method validation.

\section{Selectivity and system suitability}

Selectivity of the method was established by analyzing human plasma samples six from normal source, two from lipemic source and two from haemolysed source, to investigate the potential interferences at the LC peak region for sofosbuvir and IS. System suitability experiment was performed by injecting six consecutive injections using a standard aqueous mixture equivalent to Middle concentration of the calibration curve of sofosbuvir and IS during the start of the method validation and at the start of the each day. The percentage CV of system suitability have within the limits.

\section{Carryover effect}

Carryover effect was performed in order to eliminate the carryover from previous injection to next injection. Extracted blank sample, extracted LLOQ, and extracted ULOQ samples were prepared from same biological matrix lot. These samples were injected in the order of mobile phase, extracted blank plasma, extracted LLOQ, extracted ULOQ and extracted blank plasma samples during the start of the method validation and before using different equipment. The response of interfering peaks at the retention time of analyte have $\leq 20 \%$ of response of extracted LLOQ.

\section{Calibration curve}

Calibration curves were acquired by plotting the peak area ratios of the transition pair of sofosbuvir to that of IS against the nominal concentration of calibration standards. The concentrations used for SOF calibration curves were 30.300, 60.600, 120.905, 250.048, 500.095, 1000.190, 1500.286 and 2000.381 ng/mL, while 30.566, 80.376, 908.442 and $1700.417 \mathrm{ng} / \mathrm{mL}$ were used for LLOQ, LQC, MQC and HQC, respectively. Blank sample (without IS) and zero samples (with IS) were run with each calibration curve. The acceptance criterion for each back-calculated standard concentration was $\pm 15 \%$ deviation from the nominal value except at LLOQ, which was set at $\pm 20 \%$.

\section{Precision and accuracy}


Intra-assay precision and accuracy were estimated by analyzing six replicates containing sofosbuvir at the lower level of quantification (LLOQ) in addition to three different QC levels in human plasma. The inter-assay precision and accuracy were determined at the four levels as described above on different days. The parameter was checked on different batches and within the batches. The criteria for acceptability of the data included accuracy $\pm 15 \%$ standard deviation (SD) from the nominal values and a precision $\leq 15 \%$ coefficient of variation $\%(\mathrm{CV})$ and at LLOQ, which was set at $\pm 20 \%$.

\section{Recovery}

The extraction efficiency of sofosbuvir and IS extraction from human plasma was determined by comparing the responses of the analytes extracted from six replicate QC samples at LQC, MQC and HQC level with the response of analytes from post-extracted plasma standard sample at equivalent concentrations.

\section{Dilution integrity}

Dilution integrity was investigated to ensure that samples could be diluted with blank matrix without affecting the final concentration. Sofosbuvir spiked human plasma samples were prepared at concentrations of $8502.084 \mathrm{ng} / \mathrm{mL}$, respectively. These solutions were further diluted with pooled human plasma five folds in six replicates and analyzed. The six replicates have precision $\leq 15 \%$ and accuracy of $100 \pm 15 \%$.

\section{Ruggedness}

Ruggedness was assessed by a precision and accuracy batch. The batch was monitored using different column and different analyst.

\section{Stability experiments}

The stability of sofosbuvir and IS in the injection solvent was estimated by injecting replicate preparations of processed samples up to $30 \mathrm{~h}$ (in auto-sampler) after the initial injection. The peak areas of the analytes and IS obtained at initial cycle were used as the reference to determine the relative stability of the analytes at subsequent points. Stability of analytes in the plasma after $8 \mathrm{~h} 11$ min at room temperature (bench top) was determined at two QC concentrations (LQC and HQC) in six replicates. Freezer stability of the analytes in plasma was assessed by analyzing the QC samples (LQC, HQC and DIQC) stored at $-20 \pm 5^{\circ} \mathrm{C}$ for at least 26 days. The stability of analytes in plasma following repeated six freeze-thaw cycles (stored at $-20 \pm 5^{\circ} \mathrm{C}$ ) was assessed using QC samples (LQC and HQC) spiked with analytes. Samples were processed as described above. Samples were considered to be stable if assay values were within the acceptable limits of accuracy (i.e. $\pm 15 \%$ SD) and precision (i.e. $\leq 15 \% \mathrm{CV}$ ). 


\section{RESULTS AND DISCUSSION}

\section{Selectivity and system suitability}

The selectivity of the method was demonstrated on ten blank plasma samples obtained from different lots: the chromatograms were found to be free from endogenous significant interferences. These batches were further selected for preparation of standard CC and QC samples. The \% CV of system suitability was observed in the range of 0.20 to $0.84 \%$ for retention time of sofosbuvir and IS and 0.61 to $4.09 \%$ for area ratio, which is not more than $5.00 \%$ as per the acceptance criteria.

\section{Carryover effect}

There was no carryover observed for the interference peak obtained at the retention time (RT) of analyte is not more than or equal to $20 \%$ of extracted LLOQ and interference at the RT of ISTD is less than or equal to $5 \%$ of internal standard response of extracted LLOQ in re injected blank sample

\section{Linearity}

The optimized method was found to be linear in the concentration range of 30.300 to $2000 \mathrm{ng} / \mathrm{mL}$. A straight line was witnessed when the plot was made through the data points by $1 / \mathrm{x} 2$ weighing method and was observed to be in the range of best fit for sofosbuvir. The results found for correlation coefficient $\left(r^{2}\right)$ was greater than 0.99 as per the limits were. Thus, the method was found to be linear in the mentioned range (Table 1) and Figure (1, $2 \& 3)$.

Table: 1 Precision and Accuracy data of back-calculated concentration of calibration samples for Sofosbuvir from four representative calibration curves

\begin{tabular}{llll}
\hline $\begin{array}{c}\text { Nominal concentration } \\
(\mathbf{n g} / \mathbf{m L})\end{array}$ & $\begin{array}{l}\text { Concentration found } \\
(\mathbf{n g} / \mathbf{m L})\end{array}$ & $\begin{array}{l}\text { Precision } \\
(\mathbf{m} \mathbf{\text { RSD}})\end{array}$ & Accuracy $(\%)$ \\
\hline 30.300 & 29.711 & 1.56 & 98.06 \\
60.600 & 56.439 & 2.45 & 93.13 \\
120.905 & 109.657 & 4.04 & 90.70 \\
250.048 & 235.075 & 2.80 & 94.01 \\
500.095 & 477.527 & 5.21 & 95.49 \\
1000.190 & 1003.244 & 3.54 & 100.31 \\
1500.286 & 1462.844 & 3.57 & 97.50 \\
2000.381 & 1932.351 & 1.38 & 96.60 \\
\hline
\end{tabular}




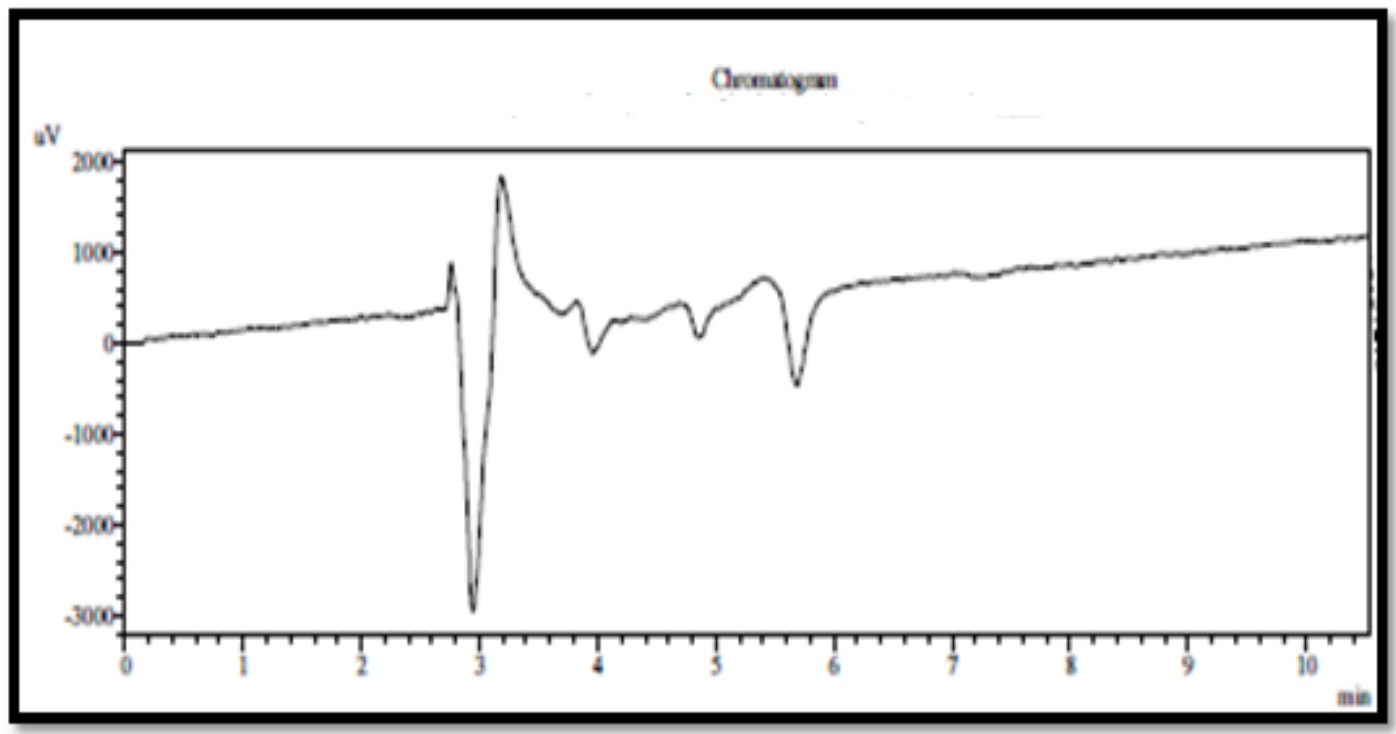

Figure 2: Extracted Blank of Sofosbuvir

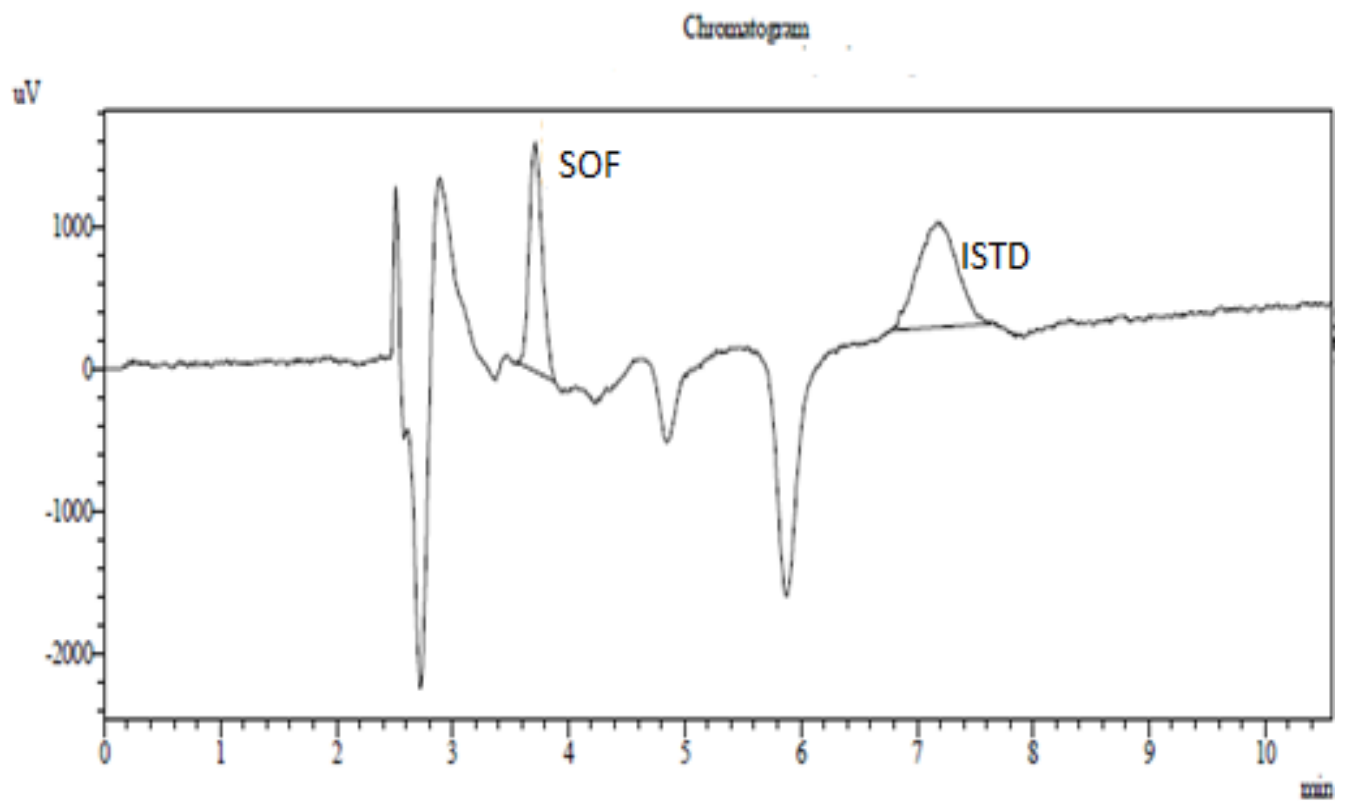

Figure 3: Extracted LLOQ of Sofosbuvir 


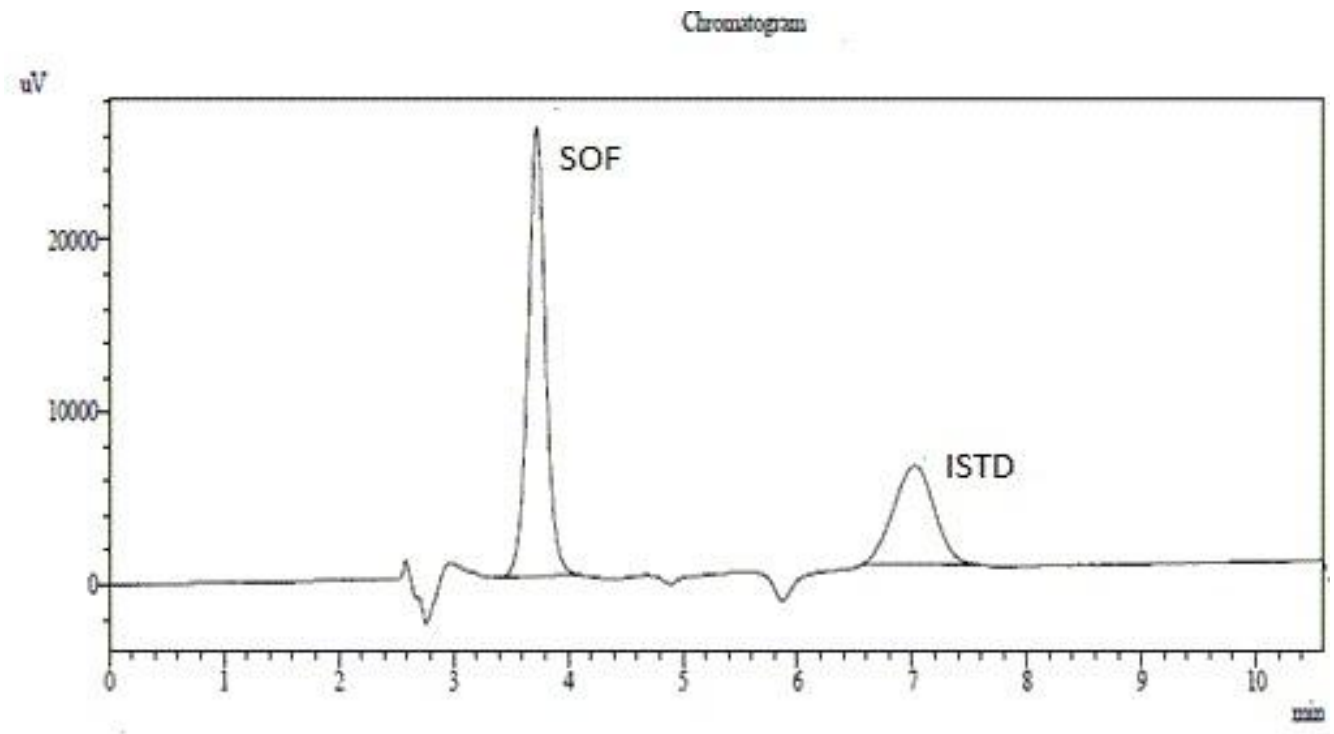

Figure 4: Extracted ULOQ of Sofosbuvir

\section{Precision and accuracy}

Precision and accuracy under inter-batch and intra-batch studies were determined and were expressed as percentage $\mathrm{CV}$ and percentage accuracy respectively. The percentage $\mathrm{CV}$ found for sofosbuvir for inter-batch QC samples were 8.70, 5.82, 5.59, and 5.01 for LLOQ, LQC, MQC and HQC respectively. Similarly, the results obtained for intra-batch QC samples ranged as 4.13-11.14, 3.45-6.49, 2.89-5.78 and 2.44-8.32 for LLOQ, LQC, MQC and HQC respectively. Accuracy in terms of percentages for sofosbuvir found under inter-batch studies was $97.21 \%, 94.97 \%, 98.38 \%$ and 97.51\% for LLOQ QC, LQC, MQC and HQC respectively. Similarly, the results obtained for intrabatch QC samples ranged as 87.99-101.87\%, 89.93-101.36\%, 90.44-103.59\% and 94.00-103.93\% for LLOQ, LQC, MQC and HQC respectively (Table 2 and 3).

Table-2 Inter-day precision and accuracy $(\mathbf{n}=24)$

\begin{tabular}{lllll}
\hline $\begin{array}{l}\text { QC } \\
\text { ID }\end{array}$ & $\begin{array}{l}\text { Nominal } \\
\text { concentration } \\
(\mathbf{n g} / \mathbf{m L})\end{array}$ & $\begin{array}{l}\text { Concentration } \\
\text { found }(\mathbf{n g} / \mathbf{m L})\end{array}$ & $\begin{array}{l}\text { Precision } \\
(\mathbf{\%} \text { RSD) }\end{array}$ & $\begin{array}{l}\text { Accuracy } \\
(\boldsymbol{\%})\end{array}$ \\
\hline LLOQ & 30.566 & 29.713 & 8.70 & 97.21 \\
LQC & 80.376 & 76.334 & 5.82 & 94.97 \\
MQC & 908.442 & 893.712 & 5.59 & 98.38 \\
HQC & 1700.417 & 1658.155 & 5.01 & 97.51 \\
DIQC & 8502.084 & 8432.230 & 7.20 & 99.18 \\
\hline
\end{tabular}


Table-3 Intra-day precision and accuracy-(n=24)

\begin{tabular}{|c|c|c|c|c|c|c|c|c|c|}
\hline \multirow[t]{2}{*}{$\begin{array}{l}\text { QC } \\
\text { ID }\end{array}$} & \multirow{2}{*}{$\begin{array}{l}\text { Nominal } \\
\text { concentration } \\
(\mathrm{ng} / \mathrm{mL})\end{array}$} & \multicolumn{4}{|c|}{$\begin{array}{l}\text { Precision } \\
\text { (\% RSD) }\end{array}$} & \multicolumn{4}{|c|}{ Accuracy (\%) } \\
\hline & & PA-01 & $\begin{array}{l}\text { PA- } \\
02\end{array}$ & $\begin{array}{l}\text { PA- } \\
03\end{array}$ & $\begin{array}{l}\text { PA- } \\
\text { 04 }\end{array}$ & $\begin{array}{l}\text { PA- } \\
01\end{array}$ & PA-02 & PA-03 & PA-04 \\
\hline LLOQ & 30.566 & 8.04 & 11.14 & 4.13 & 5.93 & 87.99 & 101.87 & 99.10 & 99.87 \\
\hline LQC & 80.376 & 3.45 & 3.92 & 6.49 & 4.20 & 89.93 & 101.36 & 96.92 & 91.67 \\
\hline MQC & 908.442 & 3.49 & 2.89 & 3.81 & 5.78 & 98.73 & 90.44 & 100.76 & 103.59 \\
\hline HQC & 1700.417 & 2.44 & 8.32 & 3.13 & 2.44 & 96.06 & 94.00 & 103.93 & 96.06 \\
\hline DIQC & 8502.084 & 5.63 & 6.53 & 2.53 & 5.71 & 96.17 & 105.93 & 92.36 & 102.26 \\
\hline
\end{tabular}

\section{Recovery}

The mean \% recovery for LQC, MQC, HQC samples of Sofosbuvir were $84.63 \%, 85.55 \%$ and $89.43 \%$ respectively. The mean overall recovery of SOF was $86.54 \%$ with a precision of $2.95 \%$ and the internal standard, mean peak area of MQC level extracted samples was compared to the mean peak area of MQC level post extracted IS and the mean \% recovery is $88.99 \%$.

\section{Dilution integrity}

Samples were analysed along with freshly spiked CC Standards and DIQC samples prepared in the screened biological matrix. The percentage accuracy was found inter-batch and intra-batch samples were $99.18 \%$ and 92.36-105.59\%. Precision percentage CV was observed inter-batch and intra-batch samples were $7.20 \%$ and $2.53-6.53 \%$.as $2.87 \%$. The results found at both the levels are within the acceptance limit.

\section{Ruggedness}

During all two cases the precision and mean accuracy for CC Standards of SOF were ranged from 2.44 to 5.93 and 91.67 to $103.59 \%$ (Table 3).

\section{Stability studies}

Stability studies were experimented and studies performed are wet extract, short time stability, long term stability, auto sampler, freeze and thaw cycles, and bench top stability. The analyte passes all stability tests viz.,Auto-injector $(30 \mathrm{~h})$, bench-top $(8 \mathrm{~h})$ and freezer stability at $-20^{\circ} \mathrm{C} \pm 5^{\circ} \mathrm{C}$ for at least for 26 days and was found unaltered to a significant extent with good percentage precision and accuracy. The freeze thaw stability results indicated that sofosbuvir was stable for six cycles. Results showed that plasma samples under investigation could be frozen and thawed without losing the reliability of the analyte under circumstances as per the optimized method (Table 4).

Table 4 Stability Samples Results for Sofosbuvir 


\begin{tabular}{|c|c|c|c|c|c|}
\hline $\begin{array}{l}\text { Stability } \\
\text { Experiment }\end{array}$ & $\begin{array}{l}\text { QC } \\
\text { ID }\end{array}$ & $\begin{array}{l}\text { Nominal } \\
\text { concentration } \\
(\mathbf{n g} / \mathbf{m L})\end{array}$ & $\begin{array}{l}\text { Concentration } \\
\text { found }(\mathrm{ng} / \mathrm{mL}) \\
(\text { mean } \pm \text { SD) }\end{array}$ & $\begin{array}{l}\text { Precision } \\
\text { (\% RSD) }\end{array}$ & $\begin{array}{l}\text { Accuracy } \\
(\%)\end{array}$ \\
\hline Bench top & LQC & 80.376 & 77.955 & 3.12 & 96.99 \\
\hline Stability & $\mathrm{HQC}$ & 1700.417 & 1637.730 & 8.34 & 96.31 \\
\hline Auto sampler & LQC & 80.376 & 79.026 & 4.93 & 98.32 \\
\hline Stability & HQC & 1700.417 & 1699.955 & 8.07 & 99.97 \\
\hline Wet extract & LQC & 80.376 & 72.689 & 3.57 & 90.44 \\
\hline Stability & HQC & 1700.417 & 1772.107 & 6.96 & 104.22 \\
\hline Freeze thaw & LQC & 80.376 & 75.221 & 3.43 & 93.59 \\
\hline Stability & HQC & 1700.417 & 1722.162 & 9.11 & 101.28 \\
\hline Long term & $\mathrm{LQC}$ & 80.376 & 81.614 & 3.76 & 101.54 \\
\hline plasma & HQC & 1700.417 & 1640.399 & 8.07 & 96.47 \\
\hline stability & DIQC & 8502.084 & 8502.823 & 5.88 & 100.01 \\
\hline
\end{tabular}

\section{CONCLUSION}

A sensitive and simple HPLC method for measurement of sofosbuvir in Human plasma has been developed and validated according to FDA Guidance for industry on bioanalytical method validation. The precision and accuracy of the method are well within the limits required for bioanalytical assays. The extraction procedure is simple, with suitable recovery. The use of the proposed method as a routine assay for pharmacokinetic, bioequivalence and drug interaction studies for the estimation of Sofosbuvir from Huma plasma.

\section{REFERENCE}

1. European Medicines Agency, Committee for Medicinal Products for Human Use (CHMP). Assessment report Sovaldi: 2017.

2. Australian Public Assessment Report for sofosbuvir. Therapeutic Good Administration: 2014 August [cited 2016 June].

3. Patent situation of key products for treatment of hepatitis C: Sofosbuvir. World Health Organization; 2016 June [cited 2016 June].

4. Saida SJ, Muniappan M, Kumar M, Kaliyaperumal M, Ramulu Y, Rao SV. Estimation of Sofosbuvir with Validated Ultra High Performance Liquid Chromatographic (UHPLC) Method in its bulk and Formulations. Der Pharmacia Sinica 2017; 8(2): 10-15.

5. Bhujbal SS, Darkunde SL. Analytical method development and optimization of sofosbuvir drug - a qbd approach. Int J Pharm Sci 2019; 10(1): 108-16.

6. Abdel S, Naby N, Gawad. Simple chromatographic and spectrophotometric determination of sofosbuvir in pure and tablet forms. Eur J Chem 2016; 7(3): 375-79. 
7. Madhavi S, Rani AP. Bioanalytical method development and validation for the determination of sofosbuvir from human plasma. Int J Pharm Pharm Sci 2017; 9(3): 35-41.

8. Vikas PM, Satyanarayana T, Kumar DV, Mounika E, Latha MS, Anusha R et al. Development and validation of new rp-hplc method for the determination of sofosbuvir in pure form. World J Pharm Pharm Sci 2016; 5(5): 775-81.

9. Mamdouh R, Emad RB, Karim BI. Development of a sensitive UPLC-ESI-MS/MS method for quantification of sofosbuvir and its metabolite, GS-331007 in human plasma: Application to a bioequivalence study. J Pharm Biomed Anal 2015; 1-36.

10. Gandhi BM, Rao AL, Rao JV. UPLC-MS/MS method for determination of sofosbuvir in human plasma. Ann Pharm Fr 2017; 75: 257-66.

11. Yang H, Robinson M, Corsa AC, Peng B, Cheng G, Tian Y et al. Preclinical Characterization of the Novel Hepatitis C Virus NS3 Protease Inhibitor GS-9451. Aac.Asm.org 2014; 58: 647-53.

12. Razeq SA, Nasr ZA, Said NS. Validated Stability-Indicating Methods for Determination of Sofosbuvir by UPLC and HPTLC in Pure Form and Tablet Dosage Forms. Asian Journal of Applied Chemistry Research 2019; 3(4): 1-13.

13. Omprakash GB, Vivek GM, Sachin BG, Wale RR, Madhuri VP. Development, validation and stability study of UV spectrophotometric method for determination of sofosbuvir in bulk and pharmaceutical dosage forms. J Pharm Res 2017;11(7): 847-49.

14. FDA. Guidance for industry: Bioanalytical Method Validation. US Department of Health and Human Services, Food and Drug Administration Centre for Drug Evaluation and Research (CDER), Centre for Veterinary Medicine (CVM): May 2001.

\section{AJPTR is}

- $\quad$ Peer-reviewed

- bimonthly

- Rapid publication

Submit your manuscript at: editor@ajptr.com

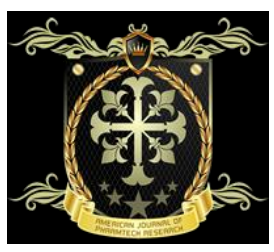

